\title{
65th AALAS National Meeting
}

\section{9-23 OCTOBER 2014}

Henry B. Gonzalez Convention Center

San Antonio, TX

Registered attendees: 4,119

Exhibiting companies: 281

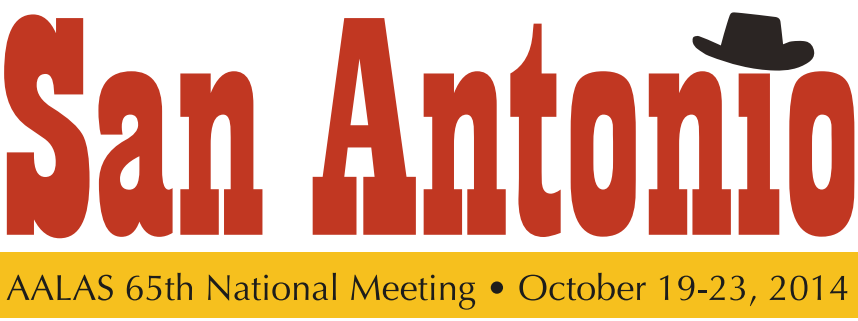

mosquito vectors and different warm-blooded hosts. After Lauren Martin (Oregon Health \& Science University, Portland) started the session with an overview of the Plasmodium life cycle, Balbir Singh (Universiti Malaysia Sarawak) explained how his group is tracking the emergence of the species $P$. knowlesi and the spread of associated infections in Malaysia. John Barnwell (US Centers for Disease Control and Prevention, Atlanta, GA) identified and compared the various nonhuman primate models used in malaria research, and Mary Galinski (Emory School of Medicine, Atlanta, GA) described the many challenges facing malaria vaccine development and how these might be addressed. Curtis Klages (University of Texas Medical Branch, Galveston) closed the session by emphasizing the biosafety concerns associated with malaria research, particularly those related to working with insect vectors in a containment laboratory.

Arthropods were not the only unusual lab animals making an appearance in the program: two special sessions centered on the care and use of non-traditional animal models. "Escaping the Shoe Box Cage: Working with Wild Animals in the Field and in the Lab," led by Jori Leszczynski (University of Colorado, Denver) on Tuesday morning, covered the challenges faced by IACUC members, veterinarians, husbandry staff members and researchers when working with wild species in biomedical research. Presenters focused on wild mammals (Robert Sikes, University of Arkansas, Little Rock), feral fish (Jill Jenkins, National Wetlands Research Center, Lafayette, LA), wild birds (Ellen Paul, The Ornithological Council, Chevy Chase, MD) and venomous and nonvenomous reptiles (Christopher Parkinson, University of Central Florida, Orlando). Thursday morning's session on "Unique Challenges Associated with Exotic Species Models," led by Sylvia Gografe (Florida Atlantic University, Boca Raton), dealt with problems like how to handle venomous snakes safely (Greg Boivin, Wright State University, Dayton, $\mathrm{OH}$ ), how to import multimammate mice captured from the wild in Africa (Rachel LaCasse, Rocky Mountain Laboratories, Hamilton, MT), how to address parthenogenesis among a lizard colony in an IACUC protocol (Diana Baumann, Stowers Institute, Kansas City, MO) and how to prevent shocks when caring for an electric eel (Rebekah Franklin, University of Wisconsin, Madison). 